\title{
Construcción de subjetividad: desde y para la comunicación digital interactiva ${ }^{11}$
}

\section{Angie Lisseth Ortega Enríquez}

Psicóloga

Universidad Pontificia Bolivariana, Colombia

Correo electrónico: angie.ortega@upb.edu.co

\section{Jimmy Jailer Quintero Valderrama}

Magister en Intervención Social Universidad Pontificia Bolivariana, Colombia

\section{Resumen}

Este artículo reflexivo enmarca la relación de la construcción de subjetividad de lo político - la política desde y para la comunicación digital interactiva. El mundo de las redes sociales ha ido recreando nuevas formas de relacionarse, comunicarse, compartir y crear contenido, saberes, comunidades, espacios y atemporalidades de interacción entre usuarios y el mundo, lo que ha llevado al sujeto a ampliar las formas de pensar, sentir y hacer. La intencionalidad de este ejercicio se ubica en esbozar la construcción de subjetividad de lo político - la política a partir del uso de las redes sociales y la necesidad de pensarse sobre el sentido y el rol de la educación en un mundo caracterizado por la singularidad, la multiplicidad, lo mutante, lo híbrido, la diferencia y lo común, la micropolítica entre otros aspectos que componen la vida y construcción del individuo, la adquisición de ideas y el ejercicio de poder.

Comunicación digital, formación, interactiva, político, política, subjetividad.

11 Para citar este artículo: Ortega, A.L. y Quintero, J.J. (2021). Construcción de subjetividad: desde y para la comunicación digital interactiva. Informes Psicológicos, 21(2), pp. 181-191 http://dx.doi.org/10.18566/infpsic.v21n2a11 


\title{
Construction of subjectivity: from and for interactive digital communication
}

\begin{abstract}
This reflective article frames the relationship of the construction of subjectivity of the politicalpolitics from and for interactive digital communication. The world of social networks has been recreating new ways of relating, communicating, sharing and creating content, knowledge, communities, spaces and timelessness of interaction between users and the world, which has led the subjects to expand their ways of thinking, feeling and doing. The intention of this exercise is to outline the construction of the subjectivity of the political-politics based on the use of social networks and the need to think about the meaning and the role of education in a world characterized by singularity, multiplicity, the mutant, the hybrid, the difference and the common, and micropolitics, among other aspects that make up the life and construction of the individual, the acquisition of ideas and the exercise of power.
\end{abstract}

Keywords

Digital communication, interactive training, the political, politics, subjectivity.

\section{Construção da subjetividade: a partir de e para a comunicação digital interativa}

\section{Resumo}

Este artigo reflexivo enquadra a relação de construção da subjetividade do político - político a partir e para a comunicação digital interativa. 0 mundo das redes sociais vem recriando novas formas de se relacionar, comunicar, compartilhar e criar conteúdos, conhecimentos, comunidades, espaços e atemporalidade de interação entre os usuários e 0 mundo, 0 que tem levado 0 sujeito a ampliar as formas de pensar, sentir e fazer. A intenção deste exercício está situada em delinear a construção da subjetividade do político-político a partir do uso das redes sociais e da necessidade de pensar 0 sentido e o papel da educação em um mundo caracterizado pela singularidade, multiplicidade, o que muda, o híbrido, a diferença e o comum, a micropolítica entre outros aspectos que compõem a vida e a construção do indivíduo, a aquisição de ideias e o exercício do poder.

\section{Palavras chave}

Comunicação digital, formação, interativa, política, política, subjetividade. 


\section{ntroducción}

Desde la llegada del sistema de distribución de información mediada por la Internet, el mundo entero asiste al progresivo y dinámico desarrollo de un nuevo orden de información caracterizado por la capacidad de converger medios de comunicación combinados, como textos, videos y sonido, que particularizan su hipertextualidad, hipermedialidad y multimedialidad (Maigret, Barbero, Lager \& Camacho, 2005); actualmente, este fenómeno ha transformado la forma desde la cual los medios de comunicación tradicionales y las personas en general transmiten la información, convirtiéndose en una interconexión con la capacidad de difundir pensamientos, gustos, acciones, contenidos y formas que sirven como espacios de socialización, producción informativa, debates e intercambios sociales y culturales.

Dicho fenómeno comunicacional contemporáneo hace alusión a una modalidad conceptual llamada ecología de la comunicación que, de acuerdo a Scolari (2015), "abarca casi todos los aspectos de los procesos comunicativos, desde las relaciones entre los medios y la economía, hasta las transformaciones perceptivas y cognitivas que sufren los sujetos a partir de su exposición a las tecnologías de la comunicación" (pp. 17-18).

Esta categoría conceptual (ecología de la comunicación) describe el contexto comunicativo de la actualidad, enmarcando el efecto que estos tienen en los sujetos que los usan, combinando, asimismo, estructura, contenido e impacto sobre los usuarios y el modo como los medios construyen la realidad (Scolari, 2015). Esta dimensión ambiental de la ecología comunicativa posibilita la emergencia de nuevas estructuras humanas, sociales, culturales y políticas implicadas en una continua construcción que va definiendo y redefiniendo formas de vida social y cultural; así, como lo menciona Aharonian (2007), "están tan imbricadas en la vida cotidiana, que nuestras formas de hacer, vivir, trabajar, consumir, relacionarnos y organizarnos se están transformando velozmente" (p.103).

En consecuencia, el nuevo panorama que trae consigo la comunicación digital e interactiva, ingresa a formar parte esencial de la interrelación de un sinnúmero de personas alrededor del mundo, generando "nuevas prácticas, lenguajes, y formas de socialización, tanto en la esfera social presencial, como en la virtual, así como nuevas formas de identificarse, representarse a sí mismos y ejercer su ciudadanía, referencian la complejización del sujeto" (Aguilar \& Said, 2010, p. 193).

Las transfiguraciones entre lo digital/ tecnológico, lo virtual/real, lo temporal/ atemporal, amplifican los efectos de este fenómeno comunicacional contemporáneo sobre la construcción de subjetividad, generando en el sujeto nuevos roles en la esfera de la comunicación e interacción, pues se establecen como activos y pasivos, consumidores y productores de contenidos distorsionando las percepciones de espacio-tiempo que modifican las formas de comunicación y establecen otras formas de acceder al conocimiento y a la experiencia humana.

En este orden, la reflexión y lectura que se comparte en este escrito sobre el fenómeno de la comunicación digital interactiva se orienta a las implicaciones de 
dicha dimensión ambiental de la ecología de la comunicación en la construcción de la subjetividad desde convergencias socioculturales que complejizan el mundo en el que habita el ser humano, un mundo caracterizado por un gran entramado social que, bajo la oportuna mirada de Castells (1999), abarca un carácter global, en donde procesos sociales y culturales como la información y la comunicación se transforman en nuevas agendas que configuran las formas de organización de una sociedad.

En efecto, este avance digital se ha convertido hoy día en un eje estratégico para el desarrollo de los procesos sociales, las convenciones a través de las cuales se adquiere el saber, y las nuevas formas y modos en las que se construye el sujeto y la subjetividad. Leer el panorama de la comunicación digital interactiva desde los diversos y múltiples contenidos que transitan por la red implica, a su vez, comprender los modos de subjetivación por los cuales atraviesa el sujeto contemporáneo, y lleva a reflexionar sobre las formas a partir de las cuales se configura lo cognitivo, lo sensorial y lo emotivo del ser humano.

\section{El escenario de la comunicación digital interactiva}

La emergencia de las tecnicidades manifestadas en las nuevas formas de información y comunicación establecen unos medios y espacios que amplían las formas desde la cual las personas acceden a la llamada sociedad de la información (Martín-Barbero, 2004). La transformación de una red mundial interconectada ha generado que la comunicación digital interactiva se esté convirtiendo en un complejo y ambiguo actor transfigurando las dimensiones psicológicas y sociales del ser humano.

Estos medios han pasado a ocupar un lugar central en la construcción de nuevas formas de percibir y de relacionarse con el mundo, con el otro y lo otro, dinamizando los procesos de información, comunicación e interacción; según la mirada de Piscitelli (2002; 2005) y Rueda (2009) es importante ubicar la reflexión y discusión sobre nuevos supuestos que amplíen la comprensión sobre la multiplicidad de factores que se encuentran articulados con la proliferación contemporánea de las nuevas tecnologías digitales, las cuales, desde sus características como la "conectividad, interactividad, usabilidad, hipertextualidad e hipermedialidad, constituyen un nuevo sistema comunicacional que transforma las prácticas, discursos, imaginarios y representaciones de la sociedad" (Scolari, 2008, p. 112).

Los atributos de este nuevo modelo de comunicación han modificado las afecciones del ser humano y la capacidad de clasificar y representar las experiencias con símbolos - signos que modifican las formas de acceder al saber, tópicos que, al tener un carácter sociocultural, modifican la dimensión de lo ético y lo político; un mundo en el que lo central transita por las formas desde las cuales las personas se comunican y se relacionan. Dichas observaciones se relacionan con la postura teórica planteada por Scolari (2008) sobre la comunicación digital interactiva, la cual refiere a "procesos de intercambio, producción y consumo simbólico que se desarrollan en un entorno caracterizado por una gran cantidad de sujetos, medios y lenguajes interconectados 
tecnológicamente de manera reticular entre sí" (pp. 113-114).

Bajo este panorama de la ecología de la comunicación, Amador (2010) menciona tres dimensiones: lo tecnológico, lo social y lo cultural, que se relacionan con los modos, procesos y dispositivos que conforman este nuevo modelo de información, comunicación e interacción generando, a su vez, producciones de lenguajes y saberes que convergen en la cultura (Igarza, 2008), y que adquieren a partir del uso y consumo una relevancia ontológica en la experiencia del sujeto $y$, por ende, en la producción de las subjetividades.

En esta lógica, el ser humano atraviesa no solo por las implicaciones de un fenómeno comunicacional contemporáneo, sino además, por las transfiguraciones de lo tecnológico y lo social, posicionándose como un nuevo modelo cultural dinamizando los procesos a partir del cual el sujeto impregna de sentido y significado su realidad, pues, hay que mencionar además, que la comunicación digital interactiva participa en matices fundamentales que hacen parte de la constitución de la subjetividad construyendo y deconstruyendo dimensiones como espacio-tiempo, construyendo diferentes experiencias de lo real (Rueda, 2009). Este nuevo panorama trae consigo la multiplicación y flexibilización entre lo real y lo virtual, el otro y lo otro.

\section{De la construcción de subjetividad}

Abordar el discurso de la construcción de la subjetividad enmarca la lectura y comprensión de una serie de espaciostiempos, discursos, prácticas, símbolos y significados que han sido cimentados en el devenir de la existencia del ser humano. Por su parte, León y Zemelman (1997) afirman que la subjetividad emerge de las disímiles realidades sociales en las que se establecen formas particulares y singulares bajo las cuales el individuo percibe, interpreta y da sentido a su realidad.

Hoy en día las dinámicas de las diferentes transformaciones tecnológicas de los nuevos medios de comunicación e información facilitan el encuentro entre sujetos, comunidades y formas de vivir, ser y pensar. Siguiendo a Clanclini (2012), caracteres como lo informativo, comunicativo e interactivo del mundo digital facilitan no solo el compartir las experiencias de los sujetos, sino también crear y recrear las formas de relacionarnos consigo mismo, con los demás y con la realidad, tópicos que participan en la construcción de los procesos de subjetivación contemporáneos.

La constitución del fenómeno psicológico en épocas contemporáneas se da bajo un nuevo ecosistema comunicacional, mediado por nuevos espacios visuales, comunicativos, transmediáticos, hipermediales e interactivos que generan, siguiendo a Amador (2010), una subjetividad a partir de la cual el sujeto halla explicación a su existencia a partir de los encuentros y desencuentros con otros, y no solo a partir de la exaltación de su propia subjetividad.

Una subjetividad que alude a una serie de acontecimientos culturales e históricos cargados de discursos y prácticas, en acuerdo con Restrepo, Urrego y García (2013), constituyen al sujeto permitiéndole 
así generar procesos de conocimiento, conciencia e importancia de múltiples experiencias simbólicas y emocionales con el fin de conceder sentido al mundo en el que vive, gestionando ciertos modos de interpretación de la realidad y de la propia existencia. El hecho de indagar por la subjetividad en épocas de transformación tecnológica, social y cultural involucra, al mismo tiempo, investigar por la conciencia, por los procesos de elaboración de significados, las emociones, los diferentes sistemas simbólicos en los que el hombre habita (Echandia, Díaz \& Vommaro, 2012). Implica, ademas, la coexistencia y la proliferación del pluralismo y la singularidad, con el fin de comprender los diferentes órdenes simbólicos en los que subyace el proceso de constitución del sujeto y su subjetividad.

Este nuevo contexto contemporáneo ha traído consigo la transformación de la comunicación digital interactiva movilizando las afecciones más íntimas del ser humano, sus experiencias y sus emociones (Blázquez, 2001). Desde este panorama, el presente trabajo propone una mirada reflexiva sobre el papel del ser humano en los fenómenos comunicacionales contemporáneos y sus efectos en la construcción de la subjetividad sobre lo político, partiendo de la comprensión ontológica que plantea Lazzarato (2006) sobre las diversas y complejas situaciones históricas, sociales y culturales que han permeado los estilos de vida que inducen al sujeto a unas formas de estar y ser en el mundo, orientando la manera de vestirse, comer, caminar, hablar, divertirse, relacionarse y aprender.

Así mismo, desde una postura crítica, Lazzarato (2006) plantea una distinción entre una democracia de la diferencia y una democracia de la multiplicidad y cómo desde cualquiera de estos dos ámbitos se da la constitución del sujeto y, por ende, de la subjetividad. Actualmente el desarrollo del sujeto se encuentra enmarcado en la relación consumidor - ciudadano, que, desde la perspectiva de Santiago (2017), se han convertido en tópicos propios de la sociedad de control que articulan las relaciones sociales y que a partir de las mismas dinámicas van construyendo un perfil de subjetividad; perfiles que se orientan según sea la democracia en la que se desarrolle el sujeto. La primera posibilidad, la democracia de la diferencia (Ríos \& Palacios, 2016), remite a la construcción de modelos mayoritarios que instalan un perfil de subjetividad con el fin de, a partir de ese universo, medir otras subjetividades, que en últimas terminan gestionando las formas y estilos de vida de los consumidores de las nuevas tecnologías de la información y la comunicación.

Dualmente, Lazzarato (2006) plantea otra organización social de Estado: la democracia de la multiplicidad, la cual se basa en la proliferación de mundos posibles conservando la singularidad del sujeto, es decir, su propio agenciamiento de vida y sus propias formas de acción. Bajo este panorama se trata entonces de comprender tres características importantes: la primera, el concepto de exterioridad, el cual se basa en descentralizar el pensamiento binario y dicotómico instalado en la época de la modernidad, y ubicar la comprensión del sujeto y la subjetividad bajo la lectura de mundos posibles, singulares y, a la vez, pluralistas.

El segundo distintivo de la democracia de la multiplicidad tiene que ver con el agenciamiento posible, el cual construye 
una plataforma de mundos posibles retomando la propuesta de las globalizaciones desde abajo (Sousa, 2003) que nutren las distribuciones de lo diverso, lo minoritario y la multiplicidad. La última particularidad reúne las características anteriores en la medida que plantea la posibilidad de habitar en un mundo posible generado y constituido por un flujo constante de expresiones, existencias, diferentes formas de ser y estar en el mundo, diversas formas de acción y de asumir la multiplicidad de las relaciones sujeto-mundo que proliferan y configuran nuevas subjetividades.

\section{Sobre lo político - la política}

Reconociendo el potencial que tienen la comunicación digital interactiva en las convergencias sociales y culturales en la constitución de la esfera pública se establece en este ejercicio de reflexión una relación entre comunicación digital interactiva, formas de socialización - subjetivación y la esfera política; así, este ejercicio enmarca, dos dimensiones: la primera, relacionada con las relaciones de poder que los sujetos establecen a partir de los vínculos e interacciones que construyen a lo largo de su vida, y la segunda, aspectos estructurales que dan cuenta de la democracia que rigen la institucionalidad y los procedimientos gubernamentales.

De acuerdo con Díaz (2003), lo político es una característica que emerge en cualquier relación humana constituyendo, al individuo desde su propia condición humana; por lo tanto, lo político desde su ejercicio aparece y se enmarca en el encuentro entre dos seres humanos. La perspectiva de la evolución humana refiere que "lo político surge cuando el primer homínido se encuentra con otro, reconociendo que ese otro no es él, pero que lo complementa, permitiéndole saber que él es el otro de otro, pero que en su complemento forman el nosotros" (Díaz, 2003, p. 50).

Esta condición de encuentro entre dos seres humanos permite en cada uno reconocer la otredad, lo diferente, lo diverso, lo minoritario, la multiplicidad y la proliferación del devenir histórico, cultural y social del otro. Dicha forma de relacionarse permite identificar que, aún bajo la diferencia, ese otro constituye y es parte importante para la existencia del otro. Se debe agregar que la formación de lo político desde la postura de Arias-Cardona y Alvarado (2015), reconoce que el ser humano depende de otros para sobrevivir, el acto de conformar familias, grupos de socialización y demás responde a la necesidad del ser humano de operar bajo una instancia que determine la vida social. Desde esta interpretación planteada por Arias-Cardona y Alvarado (2015), lo político enmarca una estrecha relación con las expresiones del sujeto, dejando ver así las formas de subjetivación en las que se ha encontrado inmerso, ya que se evidencia la contribución que algunos tiempos, espacios, sistemas simbólicos - emocionales, dispositivos e instituciones han realizado en su proceso de construcción y, además, permite evidenciar cómo estos conducen un determinado orden social bajo las diferentes relaciones sociales.

Lo anterior haciendo énfasis en que dichas relaciones e interacciones posibilitan la creación de una cadena de vínculos entre los sujetos que generan a su paso unas relaciones de poder (Ángel, 
2010), y que llevan al sujeto a un posicionamiento dentro de la sociedad en la que se encuentra inmerso. No obstante, Mouffe (1999) argumenta que lo político está unido a la particularidad de contrariedad y de discrepancia que existe en las relaciones humanas, contrariedad que se descubre como diversidad, como nuevas distribuciones de lo posible, es decir, formas diferentes, pluralistas y múltiples de estar y ser en el mundo.

Por su parte, Arendt (1997) plantea que la política presenta como características los siguientes aspectos:

La política trata del estar juntos los unos con los otros de los diversos. La política nace en el entre -los- hombres, por lo tanto, completamente fuera del hombre. De ahí que no haya ninguna substancia propiamente política. La política surge en el entre y se establece como relación (p. 45).

Dadas las consideraciones anteriores, se entiende la política como una manera desde la cual se abordan aspectos estructurales que dan cuenta de las formas de gobierno; mecanismos que rigen la institucionalidad y los procedimientos, es decir, las maneras mediante las cuales se da no solo legalidad sino también legitimidad a los aspectos anteriores, que permiten la organización y convivencia de las diferencias y pluralidades de y entre hombres y mujeres. En este sentido, la política se muestra dentro de lo político como un dispositivo adaptativo para garantizar la persistencia de la especie, bajo la usanza del poder como instrumento de control desde la institucionalidad. Desde Ángel (2010), la política se ve como "una entidad abstracta que funciona sobre la base de hacerse cargo e implica la administración de recursos desde una instancia suprema" (p. 15).

A modo de conclusiónreflexión: ¿Qué significa formar las subjetividades desde y para el contexto sociocultural en el que estamos inmersos?

El cuestionarse por la subjetividad en un tiempo en el que implícitamente emerge una nueva convergencia sociocultural, implica preguntarse por las nuevas prácticas comunicativas, las diferentes formas de relacionarse, aprender, hacer y ser del sujeto, una reflexión que parte de las múltiples transformaciones a partir de los cuales se producen los modos de subjetivación en los que se encuentra inmerso el sujeto contemporáneo, una reflexión que se posiciona desde la pregunta generadora sobre ¿qué significa formar las subjetividades desde y para el contexto sociocultural en el que estamos inmersos?

Desde el ejercicio de reflexión que se ha presentado, la dimensión desde, la cual implica indagar por la emergencia del fenómeno de la comunicación digital e interactiva que moldea nuevas configuraciones psíquicas y corporales que constituyen al sujeto. Este nuevo ecosistema comunicacional caracterizado por la transformación de la relación espaciotiempo a través de plataformas hipertextuales, interactivas, reticulares, virtuales y multimediales que le posibilitan al sujeto crear y recrear diferentes lenguajes: texto, audio, video e imagen. Dicho fenómeno hipermedial posiciona al sujeto desde un rol dicotómico entre consumidor y productor de medios y lenguajes a través de 
los cuales se relaciona, interactúa e intercambiar experiencias y percepciones del mundo, de sí mismo y del otro o lo otro.

Concluyendo este ejercicio se ubica la segunda dimensión preposicional para, la cual enmarca la indagación de las narrativas, los discursos y las relaciones de poder que moldean los afectos, los deseos y las creencias del sujeto. Dicha reflexión desde la psicología significa asumir críticamente la apropiación que hace el sujeto de las tecnologías de la comunicación y las implicaciones socioculturales de la formación del sujeto y de la subjetividad.

Desde estas nuevas formas de construir y deconstruir significados y saberes el sujeto contemporáneo ha reconfigurado las formas desde las cuales se relaciona con el otro, ha modificado sus estructuras perceptuales, lo que ha permitido ampliar su perspectiva y lectura de realidad, tópicos que conllevan a dos riesgos: el primero sobre las diversas formas de socialización y, el segundo, los posibles desequilibrios sociales. Desde este panorama, la Organización de las Naciones Unidas para la Educación, la Ciencia y la Cultura (1996) sugiere partir del principio de igualdad de oportunidades, proporcionar a los individuos las herramientas, capacidades y competencias que les permita contener la proliferación de las informaciones, seleccionarlas y jerarquizarlas con sentido crítico. Un proceso analítico que le posibilite al sujeto aprovechar la flexibilidad, multiplicidad y proliferación de la comunicación digital interactiva para plantear sus propias formas de agenciamiento de ser y estar en el mundo, ejercer los derechos y defender lo diferente y diverso.
En efecto, desde diferentes instancias de socialización del sujeto, como es el escenario académico, Hopenhayn y Ottone (2000) refieren la importancia de "equilibrar la formación en destrezas competitivas con el desarrollo del espíritu crítico, la autorreflexión y el apoyo a la cultura de pertenencia" (p. 20). Lo que permitiría, de acuerdo a Lazzarato (2006), la co-construcción de mundos posibles que requieren no solo la polifonía de lo diverso, diferente y minoritario, sino además el reconocimiento de la multiplicidad de experiencias.

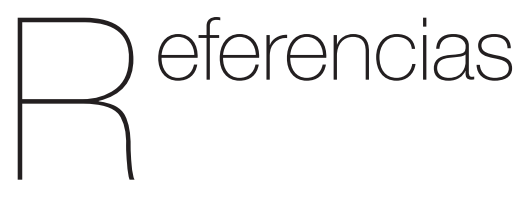

Aguilar, D. E., \& Said, E. (2010). Identidad y subjetividad en las redes sociales virtuales: caso de Facebook. Zona próxima, (12), 190-207. Recuperado de http://www. redalyc.org/html/853/85316155013/

Aharonian, A. (2007). El asesinato de la verdad. España: Editorial La Fogata

Amador, J. C. (2010). Mutaciones de la subjetividad en la comunicación digital interactiva. Consideraciones en torno al acontecimiento en los nativos digitales. Signo y Pensamiento, XXIX(57), 142-161. Recuperado de https://www.redalyc.org/ pdf/860/86020052011.pdf

Ángel, L. M. (2010). Medios de comunicación y redes virtuales, configuración de subjetividades y expresión de lo político. Estudio de caso: corre la voz (Trabajo de grado). Pontificia Universidad Javeriana. 
Bogotá, Colombia. Recuperado de https:// www.javeriana.edu.co/biblos/tesis/politica/ tesis386.pdf

Arendt, H. (1997). ¿Qué es la política? Barcelona: Editorial Paidós

Arendt, H., Cruz, M., \& Birulés, F. (1995). De la historia a la acción. Barcelona: Paidós. Recuperado de http://biblio.fcedu.uner. edu.ar/derecha/novedades/pdf/14526. PDF

Arias-Cardona, A. M., \& Alvarado, S. V. (2015). Jóvenes y política: de la participación formal a la movilización informal. Revista Latinoamericana de Ciencias Sociales, Niñez y Juventud, 13(2), 581-594. Recuperado de http://www.redalyc.org/ pdf/773/77340728003.pdf

Blázquez, F. (2001). Sociedad de la información y educación. Consejería de Educación. Ciencia y Tecnología Dirección General de Ordenación, Renovación y Centros. Mérida, España. Recuperado de http:// www.ub.edu/prometheus21/articulos/ obsciberprome/blanquez.pdf

Canclini, N. G. (2012). Jóvenes, culturas urbanas y redes digitales: prácticas emergentes en las artes, las editoriales y la música. Barcelona, España: Fundación Telefónica.

Castells, M. (1999). Internet y la sociedad Red: Internet and the network society. In Universitat Oberta de Catalunya. Recuperado de http://www.forumglobal.de/soc/bibliot/castells/ InternetCastells-htm.

Díaz, Á. (2003). Una discreta diferenciación entre la política y lo político y su incidencia sobre la educación en cuanto a la socialización política. Reflexión Política, 5(9), 48-58. Recuperado de http://www. redalyc.org/html/110/11000904/

Echandía, C. L., Gómez, Á. D., \& Vommaro, P. (Ed.). (2012). Subjetividades políticas. Bogotá, Colombia: CLACSO.

Hopenhayn, M. \& Ottone, E., (2000), El gras eslabón. Educación y desarrollo en el umbral del siglo XXI, Buenos Aires, Argentina: FCE.

Igarza, R. (2009). Burbujas de ocio. Nuevas formas de consumo cultural. Buenos Aires, Argentina: La Crujía.

Lazzarato, M. (2006). Por una política menor. Acontecimiento y política en las sociedades de control. Madrid, España: Creative Commons, Traficantes de sueños.

León, E., \& Zemelman, H. (Ed.). (1997). Subjetividad: umbrales del pensamiento social. Ciudad de México, México: Anthropos Editorial. Recuperado de https://books.google.es/books?hl=es\&lr=\& id=s4giQ1L9ObkC\&oi=fnd\&pg=PA9\&dq = Le\%C3\%B3n,+E.,+y+Zemelman,+H.+(1997 ).+Subjetividad:+umbrales+del+pensamien to+social+(Vol.+14).+Anthropos+Editorial.\& ots=CeXyollgtm\&sig=aJv1ppKjt_f3BpKfUt bvfh5Chul\#v=onepage\&q\&f=false

Maigret, É., Barbero, J.M., Lager, E., \& Camacho, E.R. (2005). Sociología de la comunicación y de los medios. México: Fondo de Cultura Económica.

Martín-Barbero, J. (2004). Tecnicidades, identidades, alteridades: desubicaciones y opacidades de la comunicación en el nuevo siglo. Guadalajara, México: Iteso. 
Mouffe, C. (1999). Deliberative de'mocracy or agonistic pluralism? Recuperado de https://www.ceeol.com/search/ article-detail?id $=157040$

Organización de las Naciones Unidas para la Educación, la Ciencia y la Cultura (1996). El Futuro del aprendizaje 2 ¿Qué tipo de aprendizaje se necesita en el siglo XXI? Recuperado de https://unesdoc.unesco. org/ark:/48223/pf0000242996_spa

Piscitelli, A. (2002). Ciberculturas 2.0: En la era de las máquinas inteligentes. Buenos Aires, Argentina: Paidós.

Piscitelli, A. (2005). Internet, la imprenta del siglo XXI. Barcelona. España: Gedisa.

Restrepo, L. R., Urrego, A. M., \& García, D. P. (2013). Sentidos entretejidos: Una aproximación a la subjetividad política en maestros y maestras de la ciudad de Medellín. Recuperado de http:// ridum.umanizales.edu.co:8080/xmlui/ handle/6789/905

Ríos, C., \& Palacios, J. (2016). Hacia una filosofía política de la diferencia. Cuestiones de Filosofia, (16), 185-197. https://doi. org/10.19053/01235095.3955

Rueda, R. (2009). Convergencia tecnológica: síntesis o multiplicidad política y cultural. Signo y Pensamiento, 28(54), 114-130. Recuperado de https://revistas.javeriana. edu.co/index.php/signoypensamiento/ article/view/4530
Santiago, A. (2017). La sociedad de control: una mirada a la educación del siglo XXI desde Foucault. Revista de Filosofía, 73, 317-336. https://dx.doi.org/10.4067/ S0718-43602017000100317

Sousa-Santos, B. (2003). La caída del Angelus Novus, ensayos para unanueva teoría social y una nueva práctica política. Universidad Nacional de Colombia. Bogotá, Colombia.

Scolari, C. (2008). Hipermediaciones: elementos para una teoría de la comunicación digital interactiva. Barcelona, España: Editorial Gedisa. Recuperado de https://books.google.es/books?hl=es\&lr= \&id=gDs|BQAAQBAJ\&oi=fnd\&pg=PA15\& $d q=$ Scolari,+C. + (2008).+Hipermediacione s:+elementos+para+una+teor\%C3\%ADa +de+la+comunicaci\%C3\%B3n+digital+in teractiva. + Editorial + Gedisa. $\&$ ots $=5 \mathrm{dpbkn}$ 16x5\&sig=VzLUeQZIHzd4veOBFs|xOXyP-

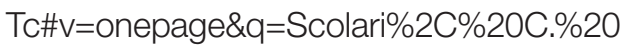
(2008).\%20Hipermediaciones\%3A\%20 elementos $\% 20$ para $\% 20$ una $\% 20$ teor \% C 3\% ADa\% 20 de\% 20la\% 20 comunicaci\%C3\%B3n\%20digital\%20 interactiva . \% $20 \mathrm{Editorial} \% 20$ Gedisa.\&f=false

Scolari, C. (2015). Ecología de los medios. Barcelona: Gedisa. 